\title{
Tissue analysis - a powerful tool for drug discovery and development
}

\author{
"In order to provide good quality data, we must understand that tissue analysis is no longer an endeavor of a single \\ person or a single functional group."
}

Keywords: compliance $=$ high-throughput $\approx$ regulatory $n$ technology $\approx$ tissue

Tissue analysis is a diverse, exciting and active subfield of bioanalysis. It is not possible to capture everything in a single issue; rather the research and review articles in this special issue offer a snapshot of the current progress and exciting developments on this topic.

Tissue analysis is not new. As early as the 1950s researchers had performed tissue analysis to study drug distribution [1] and to establish pharmacokinetic/pharmacodynamic (PK/PD) relationships [2]. Many techniques used in the 1950 s and 1960 s, such as radioactivity detection, enzymatic detection and radioimmunoassay are still being used today. So why does Bioanalysis want to put out this issue? I can think of three main reasons. First, in the 21st century, high-throughput tissue analysis is an essential feedback input, allowing frequent inspect and adapt opportunities within the drug-discovery and-development process. Therefore, there is a high value in understanding the challenges and formulating solutions to improve both the quality and throughput of tissue analysis. Second, tissue analysis has untapped potential to facilitate understanding drug actions at the cellular level. Third, we need active discussions on generating tissue analysis results for regulatory compliance.

\section{Challenges of high-quality \& high-throughput tissue analysis}

We have transitioned from discovering new drug entities via serendipity alone to systematically designing and screening bioactive compounds. To yield better drug candidates, we need to examine systematic exposure, but more importantly we need to know where the drugs are distributed in the body, whether the drug reached the target tissues at the efficacious level, and whether the drug accumulated in the tissue and reached a level that would lead to organ damage. Tissue analysis provides the experimental data to answer these questions directly. This is why we measure drug levels in tumors for oncology drugs and drug levels in the brain for CNS drugs. We also measure drugs in the liver and kidney to access potential liver toxicity or renal damage. Tissue analysis results help to weed out 'bad' compounds earlier and to identify better drug candidates.

In order to provide good quality data, we must understand that tissue analysis is no longer an endeavor of a single person or a single functional group. It is a multidisciplined endeavor. Drug metabolism and pharmacokinetic (DMPK) scientists need to identify the proper tissue(s) for analysis and interpret the resulting data. Skilled practitioners are needed to collect the tissues while maintaining the sample integrity. Input from biochemists regarding tissue collection and handling can be critical when measuring large molecular biologics and biomarkers. And evidently, bioanalytical scientists need to use properly developed methods for the tissue analysis. At the recent 13th Annual Land O'Lakes Bioanalytical Conference, I spoke about the quantitation of drugs in tissues and discussed analyses in about ten different matrices. Afterward an attendee approached me and asked whether I have experience in analyzing drugs in adipose. This encounter illustrates the point that each tissue matrix is unique in physical, chemical and biochemical properties. The drug stability and metabolic fate in those tissues are likely to be very different. Therefore, the tissue collection, handling and analytical methodologies will likely need to be different from each other. The term tissue analysis is a conglomerate - in actuality, we do brain analysis, liver analysis, heart analysis, and so on. It is rare for someone to be an expert on analysis in all tissues. But we know many brilliant scientists who are experts in analyzing drug levels in specific tissues, such as adipose, skin or eye.

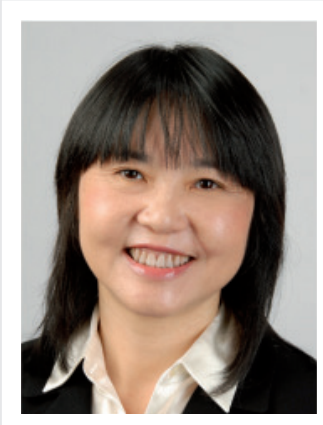

Stacy Ho

Drug Metabolism \& Pharmacokinetics, Preclinical Biosciences, Sanofi, 153 Second Avenue, Waltham, MA 0245I, USA

E-mail: stacy.ho@sanofi.com 
From the bioanalytical prospective, we face the difficulties of having no easy way to readily evaluate data quality because it is impossible to create standards and quality control samples that mimic the drug in actual tissue samples. Active research is being conducted to find better approaches to prepare standard and quality control samples, select surrogate matrices, evaluate accuracy, precision and stability, and determine recovery in tissue homogenates and in tissues.

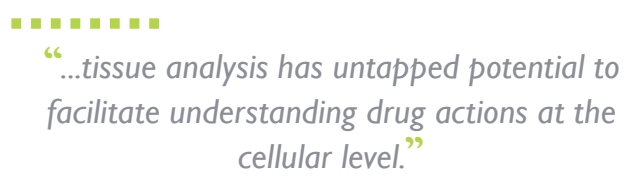

Tissue analysis throughput is important. Analyzing a mere handful of tissue samples per day is not sufficient to support the drugdiscovery engine. Currently, tissue analysis remains labor intensive mainly due to the tissue homogenization process. It is time consuming because of the need to evaluate multiple matrices during method development. Proper workflow management and the use of multiplex apparatus (e.g., Precellys-24 and FastPrep-24) and automation (e.g., Tomtec Autogizer) help, but project decisions are still too often delayed due to the lengthy time needed for analyzing tissue samples. The overall throughput of tissue analysis is in need of further improvement.

\section{Emerging technologies for tissue analysis to better understand drug actions}

Traditionally, tissue analysis yields total exposure of drugs in bulk tissues. While these results are extremely valuable, they provide neither information on drug distribution nor the concentrations of unbound drugs. Data from total drug determination in tissue homogenate sometimes generate more questions than answers, such as how does the drug distribute in the tissue? Is there any drug concentration gradient within the tissue? Does the drug penetrate the cells of target tissue or does it stay in the interstitial spaces? What is the active form of the drug (free, bound or metabolite)? In recent years, we have seen a flurry of research to tackle these questions. Most notable is the use of MS-based imaging tools, including matrixassisted laser desorption (MALDI) [3] and liquid extraction surface sampling (LESA) [4], to study the distribution of drugs within tissues.
However, most of the studies done so far are only semi-quantitative. The use of imaging technology to quantify absolute amount of drug is still very rare.

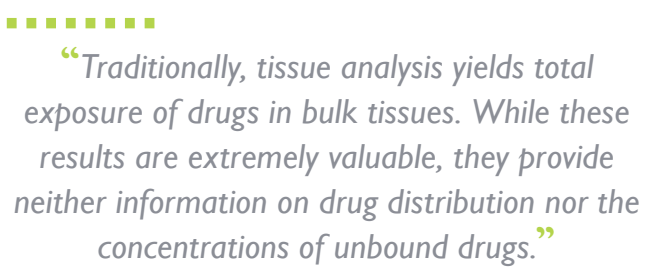
exposure of drugs in bulk tissues. While these results are extremely valuable, they provide neither information on drug distribution nor the concentrations of unbound drugs."

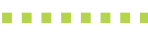

\section{Challenges of regulatory compliance}

When the results of tissue analysis are used to support regulatory action, regulatory compliance is critical [5]. We agree that we need to apply the fit-for-purpose principle [6] to demonstrate the reliability of the method. However, how should we apply that principle to tissue analysis specifically? What analytical parameters must be evaluated to ensure data integrity? Because of the tissue diversity and heterogeneity, it is unlikely to have a single answer. When we introduce high-throughput solutions to tissue analysis, such as multiplex and automation, we add new elements to the method qualification and sample analysis processes. What are the proper tests to demonstrate sample to sample consistency? How do we monitor and rectify the occasional mishaps that may occur to one of the many samples being processed together? As we adopt emerging technologies, such as using MS-based imagining tools for quantitation, we will be able to collect full scan data rather than a limited number of precursor/product ion transitions. What is the regulatory implication of this? What are the key considerations to define data for quantitation purpose? It is important to actively discuss those questions and share solutions so that we can provide regulatory compliant tissue analysis results.

Finally, I would like to thank all the contributors. Enjoy reading!

Financial \& competing interests disclosure The author has no relevant affiliations or financial involvement with any organization or entity with a financial interest in or financial conflict with the subject matter or materials discussed in the manuscript. This includes employment, consultancies, honoraria, stock ownership or options, expert testimony, grants or patents received or pending, or royalties.

No writing assistance was utilized in the production of this manuscript. 


\section{References}

1 Christensen J, Wase AW. Distribution of S35 in the Mouse after Administration of S3510(dimethylaminopropyl)-2-

chlorophenothiazine (chlorpromazine). Acta Pharmacologica et Toxicologica 12(1), 81-84 (1956).

2 Dietrich LS, Kaplan LA, Friedland IM, Martin DS. Quantitative biochemical differences between tumor and host tissue.
VI. 6-Aminonicotinamide antagonism of DPN-dependent enzymatic systems. Cancer Res. 18(11), 1272-1280 (1958).

3 Castellino S, Groseclose MR, Wagner D. MALDI imaging mass spectrometry: bridging biology and chemistry in drug development. Bioanalysis 3(21), 2427-2441 (2011).

4 Kertesz V, Van Berkel GJ. Fully automated liquid extraction-based surface sampling and ionization using a chip-based robotic nanoelectrospray platform. J. Mass Spect. 45(3), 252-260 (2010).

5 Booth B. When do you need a validated assay? Bioanalysis 3(24), 2729-2730 (2011).

6 Lee J, Devanarayan V, Barrett Y et al. Fit-forpurpose method development and validation for successful biomarker measurement. Pharm. Res. 23(2), 312-328 (2006). 